\title{
Important points in the clinical evaluation of patients with syncope
}

\author{
W Arthur, G C Kaye
}

A careful history, physical examination, and an electrocardiogram (ECG) are the most important components of the evaluation of a syncopal episode. These three components will provide a diagnosis or determine whether diagnostic testing is necessary in most patients. ${ }^{1}$ The potential cost savings made by avoiding unnecessary and expensive investigations are obvious when one considers that good clinical skills lead to the identification of the cause of syncope in $75 \%-85 \%$ of cases in which a successful diagnosis is made. ${ }^{2}$

\section{History}

A comprehensive account of the events preceding the syncopal spell is invaluable for diagnosis (box 1). The premonitory (prodromal) symptoms, precipitating factors, rate of onset, witnessed accounts, features during the recovery phase, past medical history, and the frequency and previous history of syncope will assist in the diagnosis and direct the physician in the evaluation of patients. The differential

Castle Hill Hospital, Castle Road, East Yorkshire, UK W Arthur

G C Kaye

Correspondence to: Dr Wayne Arthur,

Department of Cardiology,

Peterborough District

Hospital, Thorpe Road,

Peterborough PE3 5DE, UK

wayne@

denecres.freeserve.co.uk

Submitted 30 December

1999

Accepted 16 February 2000
Box 1: History preceding syncope

- Neurally mediated syncope

Premonitory symptoms: pallor, diaphore-

sis, nausea, visual blurring, light

headedness, hearing loss

Situation: neck turning, stress, pain, fear,

crowding, prolonged upright posture,

cough, micturition, swallowing, defecation

- Cardiac syncope

Abrupt onset, effort, or exertion, preced-

ing chest pain, palpitations

Drug related arrhythmia: antiarrhythmics, neuroleptics, digoxin, $\beta$-blockers, alcohol, illicit drugs

- Orthostatic syncope

Abrupt changes in posture

Drug related: angiotensin converting

enzyme (ACE) inhibitors, nitrates, diuret-

ics, alcohol

Postprandial

Haemorrhage
Table 1 Age distribution of syncope

\begin{tabular}{lll}
\hline Young (<35 years) & Middle aged (35-65 years) & Elderly (>65 years) \\
\hline $\begin{array}{l}\text { Neurally mediated: } \\
\text { Neurocardiogenic }\end{array}$ & Neurally mediated & Cardiac: \\
Psychiatric & Cardiac: & Arrhythmic \\
Arrhythmic & Mechanical \\
chypertrophic & Mechanical & Orthostatic: \\
[WPW syndrome] & & Drug induced \\
[Long QT syndrome] & & \\
\end{tabular}

[ ] indicates less common but important causes of syncope. WPW = Wolff-Parkinson-White. Reproduced with permission from Olshansky. ${ }^{17}$ diagnosis for syncope varies with age; this fact coupled with other historical findings can help to pinpoint the diagnosis (table 1).

\section{PRODROMAL SYMPTOMS}

Most persons with neurocardiogenic syncope have premonitory symptoms before losing consciousness; usually nausea, diaphoresis and pallor. ${ }^{3}$ Yawning, visual blurring, weakness, and a feeling of impending doom are consistent with neurally mediated syncope. The presence of palpitations preceding syncope is highly suggestive of an arrhythmia. Syncope caused by ventricular tachycardia typically occurs without a prodrome, although some patients may report brief palpitations, with light headedness preceding loss of consciousness. Supraventricular tachycardia may occasionally lead to loss of consciousness; in these rare cases palpitations are frequently noticed by the patient. ${ }^{4}$ A characteristic aura immediately preceding syncope might implicate epileptiform activity.

\section{PRECIPITATING FACTORS}

Syncope on exertion implies left ventricular outflow tract obstruction, left ventricular dysfunction, or ventricular dysrrhythmia. Loss of consciousness after the completion of exercise maybe due to a strong vagal response producing a significant bradycardia. Sudden symptom onset unrelated to posture or effort is suggestive of a cardiac arrhythmia. Coexistent sudden onset pleuritic chest pain and/or dyspnoea may indicate a pulmonary embolism. Diagnosis of carotid sinus syncope requires that spontaneous symptoms of presyncope or syncope be reproduced by carotid sinus massage. ${ }^{5}$ Correlation between symptoms and precipitating factors such as neck twisting, wearing a tight collar, shaving, or previous neck surgery reinforces the diagnosis. Syncope occurring after abrupt changes in posture would implicate orthostatic syncope. This diagnosis may frequently be missed due to concurrent hypertension in the supine position. ${ }^{6}$ Postprandial hypotension and even syncope may occur as a consequence of splanchnic shunting of blood and the intake of alcoholic beverages. ${ }^{7}$ Episodes related to stressful or emotional situations point towards neurally mediated syncope; in these circumstances the patient is often aware of losing consciousness.

\section{WITNESSED ACCOUNTS}

In addition to the history given by the patient information available from an observer of the event is crucial. The duration of syncope is by definition, transient. After a seizure loss of consciousness may be prolonged. Full assessment of the patient is not complete until eye 
witness accounts of the events preceding and following the patient's loss of consciousness are taken from those accompanying the patient to the emergency department or the clinic. If the patient is unaccompanied at initial presentation potential witnesses should be encouraged to attend as they may hold the clues to the diagnosis. It must be remembered of course that witnessed accounts, while often heavily relied upon, may be inaccurate.

RECOVERY PHASE

The account of the observer is particularly invaluable when trying to differentiate between types of syncope and seizure. The presence of confusion after the event is the single most powerful discriminator between seizure and syncope. ${ }^{8}$ Mental function is usually quickly recovered after a syncopal episode, whereas seizures are followed by a postictal period of residual confusion which can last from minutes to hours. Differentiating the recovery time between causes of syncope is more difficult but recovery after neurocardiogenic syncope can take minutes in comparison with postural hypotension or a long sinus pause after which the patient is alert within seconds. Dizziness or headache in the recovery phase (in the absence of head trauma) points towards a neurological cause.

PAST MEDICAL HISTORY

The patient with depressed left ventricular function and/or ischaemic heart disease in association with syncope is at high risk of sudden death. ${ }^{9}$ Obtaining a cardiac history and elucidating associated risk factors for ischaemic heart disease is mandatory during clinical evaluation. The presence of diabetes mellitus frequently causes silent myocardial ischaemia and may mask a myocardial infarction giving rise to a ventricular arrhythmia. Diabetic peripheral neuropathy may also lead to orthostatic syncope. Features suggestive of multiple system atrophy may be elicited from the history in patients with orthostatic syncope.

Risk can be assessed at the initial presentation by examining the frequency of syncope. If recurrent episodes are spread out over a number of years malignant arrhythmias are unlikely to be the cause. Multiple syncopal recurrences are most likely due to neurally mediated syncope or psychiatric causes. In contrast, patients with isolated $(<3)$ episodes of syncope or with a short history of recurrence are at risk of cardiac death. ${ }^{10}$ New onset syncope may herald a new serious cardiovascular cause.

DRUG HISTORY

Medications, particularly in the elderly, may be causal for syncope in a substantial number of patients. A detailed drug history should be taken; in particular changes in medication or the initiation of a new drug maybe implicated in the aetiology of the event. Antihypertensives, nitrates, and diuretics are frequently contributory to orthostatic hypotension. Vasodilatation and/or volume depletion may trigger enhanced central mechanoreceptor responses leading to neurocardiogenic syncope. Ophthalmic preparations, mostly $\beta$-blockers, can aggravate bradyarrhythmias or heart block. Antiarrhythmics, antidepressants, psychotropic drugs, many non-sedating antihistamine drugs, and other preparations may independently, or interact to, prolong the QT interval predisposing to polymorphic ventricular tachycardia.

MISCELLANEOUS POINTS

Patients with a family history of syncope or sudden death raise the possibility of the congenital long QT syndromes, hypertrophic cardiomyopathy or Wolff-Parkinson-White syndrome. There may be a familial history of neurocardiogenic syncope. A detailed family history should therefore be taken including data relating to distant relatives who died unexpectedly and any relevant familial disease. A psychiatric history may reveal anxiety disorders, major depression, and hysterical personality disorder. Patients with syncope and psychiatric disorders are younger (more often female), have a high number of syncopal episodes and have a variety of other complaints. In young patients with no structural heart disease those with multiple episodes of syncope ( $>5$ in preceding year) are less likely to have an arrhythmia and are more likely to have psychiatric illnesses. ${ }^{11}$ A full social history is also important to exclude illicit drug use as a cause of syncope and to pinpoint risk factors for ischaemic heart disease. The occupation of the patient is essential information as urgent diagnosis and therapy is required in those whose careers and safety are compromised by syncope.

\section{Physical examination}

During a thorough physical examination attention should be directed to the vital signs, the cardiovascular and neurological examination. After a supine period of at least five minutes blood pressure and pulse measurements should be taken; these should be repeated after the patient has been standing for three minutes. An abrupt drop in systolic blood pressure of between $20-30 \mathrm{~mm} \mathrm{Hg}$ with standing associated with reproduction of symptoms suggests orthostatic syncope as a cause. ${ }^{12}$ In a volume depleted patient the heart rate should rise on standing. In those with dysautonomic syncope the blood pressure can drop over several minutes while upright with no concomitant change in heart rate.

Because the prognosis after syncope is worse if there is underlying cardiac disease, the principal objective of the examination is to determine whether there is evidence for underlying cardiovascular abnormality. The cardiovascular examination may reveal a pulse character or murmurs consistent with left ventricular outflow tract obstruction. Mitral valve prolapse, left atrial myxoma, pulmonary hypertension, or prosthetic valve dysfunction may also be suspected after auscultation of the precordium. A third or fourth heart sound consistent with cardiac disease and/or congestive cardiac failure may be heard. 
In the absence of contraindications (carotid bruit, stroke, or myocardial infarction within the previous six months, or a history of ventricular tachycardia or ventricular fibrillation) carotid sinus massage (CSM) should be performed in all individuals with unexplained syncope, particularly those who are elderly with features suggestive of carotid sinus syncope..$^{13}$ Massage to the carotid sinus is performed by applying longitudinal digital pressure at the bifurcation of the internal and external carotid artery for five seconds. CSM is applied to the right then the left side after a two minute interval. Pressure should never be applied to both sides simultaneously. A standard technique of carotid sinus stimulation with applied exclusion criteria, as outlined above, causes infrequent complications most of which are transient and result in full recovery. ${ }^{14}$ Potential complications of CSM include ventricular arrhythmias and prolonged asystole, therefore continuous ECG monitoring and venous access is required.

Pulmonary examination and assessment of the jugular venous pressure may reveal congestive heart failure. The response to voluntary hyperventilation may be useful in certain patients and has been advocated as part of standard evaluation of syncope. ${ }^{15}$ In the context of an acute syncopal episode a rectal examination should be performed to look for evidence of an occult gastrointestinal bleed. The neurological examination should look to identify focal neurological signs that may offer an immediate diagnosis and redirect investigations accordingly.

\section{Electrocardiogram}

An ECG should be performed in all patients with syncope. An abnormal ECG is found in up to $50 \%$ of patients with syncope, but in most patients it is not diagnostic. ${ }^{16}$ In a person with a normal ECG there is a low likelihood that an arrhythmia is the cause of syncope, and these persons are at low risk of sudden death. ${ }^{11}$ Despite being a brief investigation the ECG together with a rhythm strip can identify potential causes of syncope in $2 \%-11 \%$ of patients. ${ }^{16}$ Acquired or congenital disease of the atrioventricular node or the distal conduction (His-Purkinje) system may be evident from second or third degree heart block. There may be evidence of conduction system disease in the His-Purkinje system with a prolonged P-R interval and associated bundle branch block and fascicular block. This pattern might implicate intermittent complete heart block as a cause of syncope and give a clue to underlying structural heart disease. The presence of bundle branch block increases the likelihood that significant sustained monomorphic ventricular tachycardia will be inducible at electrophysiological testing. ${ }^{17}$ The ECG may indicate sinus node dysfunction, myocardial infarction, ventricular pre-excitation, or a long QT interval. The electrical criteria for left ventricular hypertrophy may be fulfilled raising the suspicion of left ventricular outflow tract obstruction.

\section{Summary points}

- The history and examination are the most important stages involved in stratifying the patient into high and low risk groups and identifying the cause of syncope.

- Those with a history of structural or ischaemic heart disease or with recent onset, isolated episodes of syncope have a high risk of sudden death.

- Obtaining an eyewitness account is a vital component of the initial assessment of the cause of syncope.

- Postural hypotension and carotid sinus syncope can be reliably and safely diagnosed at the time of examination if a standard technique is adhered to.

- An abnormal ECG is found in up to $50 \%$ of patients with syncope and should therefore be part of the initial clinical assessment.

\section{Conclusion}

When a cause of syncope is determined, it is most frequently established on the basis of clinical data available at the time of initial contact with the physician. A diagnostic strategy should utilise these data as a guide to further investigation thereby minimising costly procedures that may ultimately be misleading. Such a strategy requires a thorough account of the episode(s) of syncope from the individual affected and witnesses present. The age of the patient is useful to broadly stratify causes of syncope. The routine ECG is part of the initial clinical assessment and can identify potential causes of syncope or patients with structural heart disease in significant numbers. The crux of stratifying those at high risk depends on whether or not structural heart disease is present. Gaining this information at the earliest possible opportunity should expedite the successful management of the patient.

1 Linzer $\mathrm{M}$, Yang $\mathrm{EH}$, Estes NA 3rd, et al. Diagnosing syncope. Part 1: value of history, physical examination, and electrocardiography. Clinical efficacy assessment project of the American College of Physicians. Ann Intern Med 1997;126:989-96.

2 Day SC, Cook EF, Funkenstein H, et al. Evaluation and outcome of emergency room patients with transient loss of consciousness. Am f Med 1982;73:15-23.

3 Calkins H, Shyr Y, Frumin H, et al. The value of the clinical history in the differentiation of syncope due to ventricular tachycardia, atrioventricular block, and neurocardiogenic syncope. Am f Med 1995;98:365-73.

4 Leitch JW, Klein GJ, Yee R, et al. Syncope associated with supraventricular tachycardia: an expression of tachycardia rate or vasomotor response? Circulation 1992;85:1064-71.

5 Hammill SC. Value and limitations of noninvasive assessment of syncope. Cardiol Clin 1997;15:195-218.

ment of syncope. Cardiol Clin 1997;15:195-218.
Robertson D, Robertson RM. Causes of chronic orthostatic 6 Robertson D, Robertson RM. Causes of chronic
hypotension. Arch Intern Med 1994;154:1620-4.

7 Lipsitz LA, Ryan SM, Parker A, et al. Hemodynamic and autonomic nervous system responses to mixed meal ingestion in healthy young and old subjects and dysautonomic patients with postprandial hypotension. Circulation 1993;87:391-400.

8 Gastaut H, Fischer-Williams M. Electroecephalographic study of syncope: its differentiation from epilepsy. Lancet 1957;ii: $1018-25$

9 Middlekauff HR, Stevenson WG, Saxon LA. Prognosis after syncope: impact of left ventricular function. Am Heart $\mathcal{F}$ 1993;125:121-7.

10 Krol RB, Morady F, Flaker GC, et al. Electrophysiological testing in patients with unexplained syncope: clinical noninvasive predictors of outcome. $\mathcal{F} \mathrm{Am}$ Coll Cardiol 1987;10: vasive pred $358-63$.

11 Kapoor WN. Diagnostic evaluation of syncope. Am $\mathcal{F}$ Med 1991;90:91-106. 
12 Atkins D, Hanusa B, Sefcik C, et al. Syncope and orthostatic hypotension. Am f Med 1991;91:179-85.

13 Wagshal AB, Huang SKS. Carotid sinus hypersensitivity. In: Grubb BP, Olshansky B, eds. Syncope: mechanisms and management. Armonk, NY: Futura, 1998: 281-95.

14 Davies AJ, Kenny RA. Frequency of neurologic complications following carotid sinus massage. Am $\mathcal{f}$ Cardiol 1998;81:1256-7.
15 Linzer M, Varia I, Pontinen M, et al. Medically unexplained syncope: relationship to psychiatric illness. Am f Med 1992; 92:18-25.

16 Kapoor WN. Evaluation and outcome of patients with syncope. Medicine 1990;69:160-75.

17 Olshansky B. Syncope: overview and approach to management. In: Grubb BP, Olshansky B, eds. Syncope: mechanisms and management. Armonk, NY: Futura, 1998: 15-71. 